\title{
Avaliação do nível de conhecimento dos estudantes de odontologia do centro universitário de João Pessoa em relação à prescrição de medicamentos
}

Evaluation of level of knowledge of students of dentistry center college of João Pessoa in relation to prescription drugs Evaluación del nivel de conocimiento de los estudiantes de odontología del centro universitario de João Pessoa en relación a la prescripción de medicamentos

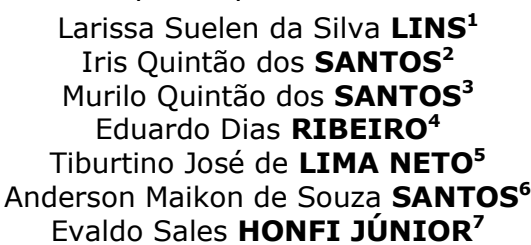

${ }^{l}$ Graduanda em Odontologia pela Universidade Federal da Paraíba UFPB, João Pessoa-PB, Brasil ${ }^{2}$ Cirurgiã-Dentista pelo Centro Universitário de João Pessoa UNIPÊ, João Pessoa-PB, Brasil ${ }^{3}$ Cirurgião Bucomaxilofacial pelo Hospital Universitário Laura Wanderley HULW

${ }^{4}$ Professor Adjunto do Curso de Odontologia da Universidade Federal da Paraíba (UFPB) João Pessoa-PB, Brasil

${ }^{5}$ Mestrando, Programa de Pós-Graduação em Odontologia, área de concentração Cirurgia e Traumatologia Bucomaxilofacial, Universidade Estadual Paulista (UNESP) Faculdade de Odontologia de Araçatuba, Araçatuba-SP, Brasil

${ }^{6}$ Mestrando, Programa de Pós-Graduação em Odontologia, área de concentração Cirurgia e Traumatologia Bucomaxilofacial, Universidade Estadual Paulista (UNESP) Faculdade de Odontologia de Araçatuba, Araçatuba-SP, Brasil

${ }^{7}$ Professor Assistente da Disciplina de Cirurgia Oral e Maxilofacial I e II do Curso de Odontologia do Centro Universitário de João Pessoa (UNIPÊ), Professor de Anatomia Bucomaxilofacial do Curso de Odontologia do Instituto de Educação Superior da paraíba (IESP), João Pessoa-PB, Brasil

\section{Resumo}

Introdução: A prescrição de medicamentos é uma ordem escrita e dirigida ao farmacêutico, que defini como o fármaco deve ser dispensado para o paciente. Objetivo: Analisar qual o nível de conhecimento dos estudantes de odontologia do Centro Universitário de João Pessoa UNIPÊ sobre prescrição de medicamentos. Metodologia: Foi realizada uma pesquisa quantitativa e descritiva com alunos do $5^{\circ}$ ao $10^{\circ}$ período do curso de Odontologia do Centro Universitário de João Pessoa - UNIPÊ, localizado na Br 230, Km 22, Água Fria, João Pessoa, Paraíba. Foi utilizado como instrumento de coleta de dados desta pesquisa um questionário, estruturado e elaborado pelo aluno pesquisador e pelo professor orientador com base na fundamentação teórica estudada. O questionário é composto por 12 questões objetivas. Os dados da pesquisa foram coletados e após, analisados pelo Programa Statistical Package for the Social Sciences (SPSS) versão 13.0. Resultados: A maior parte da amostra correspondeu ao gênero feminino, tendo entre 15 e 25 anos, solteiros e que se sentiam seguros em prescrever, a maioria não conhecem os tipos de medicamentos que por eles possam ser prescritos nem a lista que oferece ao profissional base de uma prescrição segura elaborada pelo Sistema Único de Saúde, que é a Relação Nacional de Medicamentos essenciais (RENAME). Conclusão: Ainda é baixo o nível de conhecimento dos estudantes de odontologia do Centro Universitário de João Pessoa em relação a os medicamentos contidos na lista de medicamentos essenciais (RENAME) do Sistema Único de Saúde (SUS).

Descritores: Sistema Único de Saúde; Farmacologia; Estudantes de Odontologia.

\section{Abstract}

Introduction: The prescription of medicines is a written order and addressed to the pharmacist, which defined how the drug should be dispensed for the patient. Objective: To analyze the level of knowledge of dentistry students of the University Center of João Pessoa -

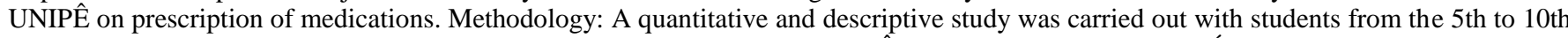
period of the Dentistry course of the University Center of João Pessoa - UNIPÊ, located at Br 230, Km 22, Água Fria, João Pessoa, Paraíba. It was used as a data collection instrument of this research a questionnaire, structured and elaborated by the student researcher and the guiding teacher based on the theoretical foundation studied. The questionnaire consists of 12 objective questions. The survey data were collected and then analyzed by the Statistical Package for Social Sciences (SPSS) version 13.0. Results: The majority of the sample corresponded to the female gender, between 15 and 25 years old, who were unmarried and who felt safe to prescribe, most do not know the types of drugs that can be prescribed by them or the list that offers the professional base of a safe prescription elaborated by the Unified Health System, which is the National List of Essential Medicines (RENAME). Conclusion: The level of knowledge of the dentistry students of the University Center of João Pessoa is still low in relation to the medicines contained in the list of essential medicines (RENAME) of the Unified Health System (SUS).

Descriptors: Unified Health System; Pharmacology; Students, Dental

\section{Resumen}

Introducción: La prescripción de medicamentos es una orden escrita y dirigida al farmacéutico, que define como el fármaco debe ser dispensado para el paciente. Objetivo: Analizar el nivel de conocimiento de los estudiantes de odontología del Centro Universitario de João Pessoa - UNIPÊ sobre prescripción de medicamentos. Metodología: Se realizó una investigación cuantitativa y descriptiva con alumnos del $5^{\circ}$ al $10^{\circ}$ período del curso de Odontología del Centro Universitario de João Pessoa - UNIPÊ, ubicado en la Br 230, Km 22, Agua Fría, João Pessoa, Paraíba. Se utilizó como instrumento de recolección de datos de esta investigación un cuestionario, estructurado y elaborado por el alumno investigador y por el profesor orientador con base en la fundamentación teórica estudiada. El cuestionario se compone de 12 cuestiones objetivas. Los datos de la encuesta fueron recolectados y después, analizados por el Programa Statistical Package for the Social Sciences (SPSS) versión 13.0. Resultados: La mayor parte de la muestra correspondió al género femenino, entre 15 y 25 años, solteros y que se sentían seguros en prescribir, la mayoría no conocen los tipos de medicamentos que por ellos puedan ser prescritos ni la lista que ofrece al profesional base de una prescripción segura elaborada por el Sistema Único de Salud, que es la Relación Nacional de Medicamentos esenciales (RENAME). Conclusión: Aún es bajo el nivel de conocimiento de los estudiantes de odontología del Centro Universitario de João Pessoa en relación a los medicamentos contenidos en la lista de medicamentos esenciales (RENAME) del Sistema Único de Salud (SUS).

Descriptores: Sistema Único de Salud; Farmacología, Estudiantes de Odontologia

\section{INTRODUÇÃO}

Prescrição de medicamento é uma ordem escrita dirigida ao farmacêutico, que defini como o fármaco deve ser dispensado ao paciente, determinando as condições em que o medicamento deve ser utilizado e apresentado por escrito, pois é de inteira responsabilidade tanto de quem prescreve, quanto de quem dispensa ${ }^{1}$.

No Brasil, a disponibilização de 
medicamentos adequadamente às necessidades da população ainda é um desafio a ser superada, apesar da implantação de políticas públicas na década de 1990, a Política Nacional de Medicamentos, a Política Nacional de Assistência Farmacêutica, a Política de Medicamentos Genéricos e o Programa Farmácia Popular ${ }^{2}$. Além disso, o gasto privado na aquisição de remédios é muito grande no país ${ }^{3}$, ainda ao se considerar a existência de um sistema público de saúde, com cobertura universal e sem pagamento direto dos usuários. $\mathrm{O}$ acesso insuficiente aos medicamentos está diretamente associado com piora do estado de saúde, o maior uso de terapias adicionais, como o uso de quantidade excessiva de medicamentos, o aumento no número de retornos aos serviços de saúde e gastos adicionais nos tratamentos ${ }^{4}$.

Os cirurgiões-dentistas devem conhecer as vias de administração e a ação dos medicamentos que por eles possam ser prescritos, devendo também analisar criticamente a bibliografia pesquisada e as bulas oferecidas pelos laboratórios farmacêuticos, e também analisar os resultados apresentados pelo uso dos medicamentos.

A interação de medicamentos é um evento em que os efeitos de um fármaco ficam alterados pela presença de outro fármaco, alimento, bebida alcoólica ou agente químico ambiental ${ }^{5}$.

Desta forma, este trabalho tem o objetivo de avaliar qual o nível de conhecimento dos estudantes de odontologia do centro universitário de João Pessoa em relação à prescrição de medicamentos. $\mathrm{O}$ propósito deste trabalho foi avaliar o conhecimento dos estudantes de Odontologia do Centro Universitário de João Pessoa, UNIPÊ em relação prescrição de medicamentos, com foco nas medicações disponibilizadas pelo Sistema Único de Saúde - SUS e na atualização de conhecimentos na área de terapêutica medicamentosa.

\section{MATERIAL E MÉTODO}

Este estudo foi classificado como quantitativo, exploratório, descritivo e bibliográfico. Segundo Silva e Menezes ${ }^{6}$ quantitativa porque considera que tudo pode ser quantificavel, o que significa traduzir em números opiniões e informações para classificá-los e analisá-lo, requerendo o uso de recursos e de técnicas estatísticas.

De acordo com $\mathrm{Gil}^{7}$, pesquisa exploratória tem como objetivo proporcionar maior familiaridade com o problema, com vistas a torná-lo mais explícita ou a constituir hipóteses e a pesquisa descritiva procura observar, registrar, analisar, classificar, e interpretar os fatos ou fenômenos sem que o pesquisador interfira neles.

- Campo da Pesquisa

A pesquisa, aprovada junto ao Comitê de Ética em Pesquisa Centro Universitário de João
Pessoa (UNIPE)/CAAE 19239313.5.0000.5176 foi realizada no Curso de Odontologia do Centro Universitário de João Pessoa - UNIPÊ, localizado na $\mathrm{Br} 230, \mathrm{Km} 22$, Água Fria, João Pessoa, Paraíba.

- Universo e Amostra

O universo deste estudo foi representado pelos 300 alunos regularmente matriculados do $5^{\circ}$ ao $10^{\circ}$ período do Curso de Odontologia do Centro Universitário de João Pessoa - UNIPÊ; a amostra do estudo foi de 159 estudantes.

\section{- Critérios de inclusão}

Alunos de ambos os sexos, com 18 anos ou mais, regularmente matriculados do $5^{\circ}$ ao $10^{\circ}$ período no Curso de Odontologia do Centro Universitário de João Pessoa - UNIPÊ, que concordem em participar da pesquisa e assinem o termo de consentimento livre e esclarecido;

\section{- Critérios de exclusão}

Alunos que não se sintam aptos a preencher o questionário proposto na pesquisa ou que deixem de responder alguma questão, além dos alunos que não queiram assinar o termo de consentimento livre e esclarecido.

\section{- Instrumento de Coleta de Dados}

Foi utilizado como instrumento de coleta de dados questionário estruturado composto por 12 questões objetivas, atendendo aos objetivos da pesquisa.

\section{- Procedimentos Metodológicos}

Após explanação da pesquisa aos estudantes do Curso de Odontologia do Centro Universitário de João Pessoa (UNIPÊ), expondo benefícios, riscos, desconfortos e confidencialidade do experimento, os voluntários que aceitaram participar assinaram o Termo de Consentimento Livre e Esclarecido (apêndice A). Na sequência, responderam ao questionário contendo 12 questões objetivas para coleta dos dados.

\section{- Tratamento dos Dados}

Os dados da pesquisa foram coletados, analisados pelo Programa Statistical Package for the Social Sciences (SPSS) versão 13.0 e transformados em percentuais, utilizando-se tabelas e gráficos para melhor entendimento dos resultados colhidos.

\section{RESULTADOS E DISCUSSÃO}

No presente estudo foram entrevistados 159 acadêmicos do $5^{\circ}$ período ao $10^{\circ}$ período do Curso de Odontologia do Centro Universitário de João Pessoa - UNIPÊ para verificar o conhecimento dos estudantes em relação à prescrição de medicamentos.

As Universidades devem ser a base para a formação de recursos humanos da área de saúde, buscando qualificação para a eficiência e eficácia dos serviços. Supõe-se ser a formação acadêmica a fonte inicial de conhecimentos terapêuticos para a correta prescrição medicamentosa ${ }^{8}$.

A maioria dos acadêmicos do Curso de 
Odontologia do Centro Universitário de João Pessoa (UNIPÊ) é composta por estudantes do gênero feminino em 68,6\% (Tabela 1).

Tabela 1. Distribuição dos alunos do Curso de Odontologia do Centro Universitário de João Pessoa (UNIPE) segundo o sexo

\begin{tabular}{l|c|c}
\hline \multirow{2}{*}{ Sexo } & \multicolumn{2}{c}{ Frequência } \\
\cline { 2 - 3 } & n & \% \\
\hline Masculino & 50 & 31,4 \\
\hline Feminino & 109 & 68,6 \\
\hline Total & $\mathbf{1 5 9}$ & $\mathbf{1 0 0}$ \\
\hline Fonte: Dados da pesquisa.
\end{tabular}

Concordando com os resultados colhidos por Araújo ${ }^{9}$ em pesquisa realizada na Faculdade de Odontologia de Ribeirão Preto na Universidade de São Paulo (USP), relatando uma prevalência de cerca de 70,0\% dos estudantes de Odontologia do gênero feminino. Os dados obtidos também corroboram estudos de Costa et al. ${ }^{10}$ realizado Universidade Estadual de Montes Claros (UNIMONTES), avaliando o percentual de alunos do gênero feminino inscritos no vestibular para o Curso de Odontologia no período de 1999 a 2006, obtendo 65,1\%.

Quando analisada a faixa etária, na pesquisa realizada com os graduandos de Odontologia do Centro Universitário de João Pessoa (UNIPÊ) encontrou-se $82,4 \%$ dos participantes com idade entre 15 e 25 anos (Tabela 2).

Tabela 2. Distribuição dos alunos do Curso de Odontologia do Centro Universitário de João Pessoa (UNIPE) segundo a faixa etária

\begin{tabular}{l|c|c}
\hline \multirow{2}{*}{ Faixa Etária } & $\mathbf{2}$ & Frequência \\
\cline { 2 - 3 } 15 a 25 anos & 131 & 82,4 \\
\hline 26 a 35 anos & 26 & 16,4 \\
\hline 36 a 45 anos & 1 & 0,6 \\
\hline Mais de 45 anos & 1 & 0.6 \\
\hline Total & $\mathbf{1 5 9}$ & $\mathbf{1 0 0}$ \\
\hline Fonte: Dados da pesquisa.
\end{tabular}

Concordando com os resultados colhidos Sundefeld e et al. ${ }^{11}$ que realizaram pesquisa com ingressantes do Curso de Odontologia da Faculdade de Odontologia de Araçatuba (UNESP), onde foram entrevistados 527 acadêmicos, em um período de 2005 a 2009, obtendo-se idade média 18,99 anos. Já Brustolin $^{12}$ em estudo conduzido na Universidade do Planalto Catarinense, no qual foram entrevistados 214 acadêmicos, observando-se faixa etária dos alunos de Odontologia variando entre 16 e 41 anos com média de 20 a 21 anos.

Os resultados colhidos na presente pesquisa apontam que a maior parte dos alunos de Odontologia do Centro Universitário de João Pessoa (UNIPÊ) é solteiro $(81,8 \%)$ (Tabela 3). Resultados semelhantes foram obtidos por Sullcahuamán ${ }^{13} \mathrm{em}$ estudo realizado no Departamento de Odontologia na Universidade Federal do Paraná (UFPR) onde 96,2\% de estudantes eram solteiros. Brustolin, et al. ${ }^{12}$ também encontraram valor bem próximo ao avaliar o perfil dos acadêmicos de Odontologia da Universidade do Planalto Catarinense (UNIPLAC), onde $90,7 \%$ eram solteiros.

Tabela 3. Distribuição dos alunos do Curso de Odontologia do Centro Universitário de João Pessoa (UNIPE) segundo o estado civil

\begin{tabular}{l|c|c}
\hline \multirow{2}{*}{ Estado Civil } & $\mathbf{2}$ Frequência \\
\cline { 2 - 3 } & 130 & $\mathbf{\%}$ \\
\hline Solteiro & 26 & 81,8 \\
\hline Casado & 2 & 16,3 \\
\hline Viúvo & 1 & 1,3 \\
\hline Divorciado & $\mathbf{1 5 9}$ & $\mathbf{1 0 0}$ \\
\hline Total & \multicolumn{2}{|}{} \\
\hline Fonte: Dados da pesquisa. &
\end{tabular}

Dos alunos de Odontologia do Centro Universitário de João Pessoa (UNIPÊ) avaliados na presente pesquisa, $67,9 \%$ relataram que estavam seguros em prescrever para o seu paciente (Tabela 4), contrastando com os dados obtidos por Britto et al. ${ }^{8}$ na Faculdade de Odontologia da Universidade Federal de Minas Gerais (UFMG) e no Departamento de Odontologia da Pontifica Universidade Católica de Minas Gerais (PUCMG), os quais apontaram insegurança dos estudantes na prescrição de medicamentos. Uma possível explicação, segundo os autores ${ }^{8}$ seria o fato da disciplina de farmacologia ser ministrada no ciclo básico e a sua continuação, como conteúdo auxiliar das disciplinas práticas, não ser sistematizada. Para Castilho et al. $^{14}$, que avaliou a prescrição de medicamentos de uso sistêmico por cirurgiõesdentistas clínicos gerais, $30 \%$ da amostra não considera a farmacologia muito importante na sua vida profissional e $44,8 \%$ dos pesquisados consideram insuficientes os seus conhecimentos nesta área.

Tabela 4. Distribuição dos alunos do Curso de Odontologia do Centro Universitário de João Pessoa (UNIPE) segundo a segurança na prescrição de medicamentos

\begin{tabular}{l|c|c}
\hline \multirow{2}{*}{$\begin{array}{c}\text { Segurança na } \\
\text { prescrição }\end{array}$} & $\mathbf{n}$ & Frequência \\
\cline { 2 - 3 } & 108 & 67,9 \\
\hline Sim & 51 & 32,1 \\
\hline Não & $\mathbf{1 5 9}$ & $\mathbf{1 0 0}$ \\
\hline Total & \multicolumn{2}{|}{} \\
\hline Fonte: Dados da pesquisa. &
\end{tabular}

Cerca de 67,9\% dos graduandos do Centro Universitário de João Pessoa (UNIPÊ) relatou ter frequentado cursos extracurriculares na área de farmacologia e prescrição de medicamentos (Tabela 5). Em estudo realizado por Marmitt ${ }^{15}$ na Faculdade de Odontologia da Universidade Federal do Rio Grande do Sul (UFRS) os entrevistados foram questionados sobre as fontes de seus conhecimentos sobre a prescrição de medicamentos. Segundo o autor, apenas $10 \%$ relataram terem participado de cursos extracurriculares sobre o tema. A maioria (47\%) afirmou que no curso de graduação iria aprender o necessário sobre uma prescrição correta; 
$26 \%$ apontaram que tinham aprendido por interesse próprio e $15 \% \mathrm{em}$ rotinas dos serviços de atendimento. Castilho et al. ${ }^{14}$, em estudo com 163 cirurgiões-dentistas da cidade de Belo HorizonteMG, observaram que $78 \%$ haviam participado de cursos de reciclagem em farmacologia tanto na graduação, quanto após formados.

Tabela 5. Distribuição dos alunos do Curso de Odontologia do Centro Universitário de João Pessoa (UNIPE) segundo a participação em cursos extracurriculares

\begin{tabular}{l|c|c}
\hline \multirow{2}{*}{$\begin{array}{c}\text { Participação em } \\
\text { cursos } \\
\text { extracurriculares }\end{array}$} & $\mathbf{n}$ & $\mathbf{2}$ \\
\cline { 2 - 3 } Sim & 108 & 67,9 \\
\hline Não & 51 & 32,1 \\
\hline Total & $\mathbf{1 5 9}$ & $\mathbf{1 0 0}$ \\
\hline Fonte: Dados da pesquisa. & \multicolumn{2}{|}{}
\end{tabular}

$\mathrm{Na}$ pesquisa realizada com os alunos do Centro Universitário de João Pessoa (UNIPÊ) foi observado que $74,8 \%$ acreditam que apenas médico e cirurgião-dentista podem prescrever medicamentos para os seus pacientes (Tabela 6).

Britto et al. ${ }^{8}$ lembram que de acordo com a legislação vigente, tanto médico, como o cirurgiãodentista e o médico veterinário (este na área animal) podem prescrever medicamentos que julgarem mais adequados para curar, diminuir ou estabilizar as enfermidades diagnosticadas em seus pacientes.

Tabela 6. Distribuição dos alunos do Curso de Odontologia do Centro Universitário de João Pessoa (UNIPE) segundo profissionais considerados habilitados para a prescrição de medicamentos

\begin{tabular}{l|c|c}
\hline \multirow{2}{*}{$\begin{array}{c}\text { Profissionais habilitados } \\
\text { para a prescrição de } \\
\text { medicamentos }\end{array}$} & $\mathbf{2}$ & $\mathbf{2}$ \\
\cline { 2 - 3 } & 3 & 1,9 \\
\hline Cirurgião-Dentista & 1 & 0,6 \\
\hline Médico & 120 & 74,8 \\
\hline Médico e Cirurgião-Dentista & 1 & 0,6 \\
\hline Médico e Psicólogo & 9 & 5,7 \\
\hline $\begin{array}{l}\text { Médico, Cirurgião-Dentista e } \\
\text { Enfermeiro }\end{array}$ & 3 & 1,9 \\
\hline $\begin{array}{l}\text { Médico, Cirurgião-Dentista e } \\
\text { Psicólogo }\end{array}$ & 8 & 5,0 \\
\hline $\begin{array}{l}\text { Médico, Cirurgião-Dentista e } \\
\text { Médico Veterinário }\end{array}$ & 12 & 7,5 \\
\hline $\begin{array}{l}\text { Médico, Cirurgião-Dentista e } \\
\text { Farmacêutico }\end{array}$ & 1 & 0,6 \\
\hline $\begin{array}{l}\text { Médico, Cirurgião-Dentista, } \\
\text { Farmacêutico e Psicólogo }\end{array}$ & 1 & 0,6 \\
\hline $\begin{array}{l}\text { Médico, Cirurgião-Dentista, } \\
\text { Farmacêutico e Enfermeiro }\end{array}$ & $\mathbf{1 5 9}$ & $\mathbf{1 0 0}$ \\
\hline Total &
\end{tabular}

A maioria alunos do Centro Universitário de João Pessoa (UNIPÊ) participante da pesquisa $(41,2 \%)$ acreditam que os cirurgiões-dentistas podem prescrever somente antibióticos, analgésicos, antifúngicos, antiinflamatórios esteróides e antiinflamatórios não-esteroides (Tabela 7).

Para Garbin. et al. ${ }^{16}$ os cirurgiões-dentistas podem prescrever um pequeno arsenal de drogas, mas, frequentemente, limitam-se aos antimicrobianos, analgésicos e anti-inflamatórios. Entretanto, é importante ressaltar que a Agência Nacional de Vigilância Sanitária (ANVISA) ${ }^{17}$ obrigatoriamente preconiza que qualquer medicamento de marca, similar ou genérico, exceto os de venda livre, deverá ser comercializado mediante prescrição farmacológica.

Tabela 7. Distribuição dos alunos do Curso de Odontologia do Centro Universitário de João Pessoa (UNIPE) segundo os medicamentos da alçada de prescrição do cirurgião-dentista

\begin{tabular}{|c|c|c|}
\hline \multirow{2}{*}{$\begin{array}{l}\text { Medicamentos que podem ser } \\
\text { prescritos pelo cirurgião-dentista }\end{array}$} & \multicolumn{2}{|c|}{ Frequência } \\
\hline & $\mathbf{n}$ & $\%$ \\
\hline $\begin{array}{l}\text { Analgésicos } \\
\text { esteroides }\end{array}$ & 1 & 0,6 \\
\hline Analgésicos, antifúngicos e AINES & 1 & 0,6 \\
\hline Antibióticos e analgésicos & 10 & 6,3 \\
\hline $\begin{array}{l}\text { Antibióticos, analgésicos, antifúngicos } \\
\text { e AINES }\end{array}$ & 1 & 0,6 \\
\hline $\begin{array}{l}\text { Antibióticos, analgésicos, antifúngicos, } \\
\text { anti-inflamatórios esteroides e AINES }\end{array}$ & 71 & 41,2 \\
\hline Antibióticos, analgésicos e antifúngicos & 10 & 6,3 \\
\hline $\begin{array}{l}\text { Antibióticos, analgésicos, } \\
\text { anti-inflamatórios esteroides e AINES }\end{array}$ & 9 & 5,6 \\
\hline Antibióticos, analgésicos e AINES & 1 & 3,4 \\
\hline $\begin{array}{l}\text { Antibióticos, analgésicos } \\
\text { inflamatórios esteroides }\end{array}$ & 4 & 2,5 \\
\hline $\begin{array}{l}\text { Antibióticos, analgésicos, antifúngicos } \\
\text { e AINES }\end{array}$ & 3 & 1,9 \\
\hline $\begin{array}{l}\text { Antibióticos, analgésicos, antifúngicos } \\
\text { e anti-inflamatórios esteroides }\end{array}$ & 1 & 0,6 \\
\hline $\begin{array}{l}\text { Nimesulida, diclofenaco de sódio, } \\
\text { amoxicilina, dipirona, paracetamol, } \\
\text { torsilax, buscopan e transamin }\end{array}$ & 35 & 21,9 \\
\hline $\begin{array}{ll}\text { Antibióticos, } \quad \text { analgésicos } & \text { anti- } \\
\text { inflamatórios esteroides e AINES } & \end{array}$ & 12 & 7,9 \\
\hline Total & 159 & 100 \\
\hline
\end{tabular}

Para a maior parte dos acadêmicos de Odontologia do Centro Universitário de João Pessoa (UNIPÊ) apenas antibióticos $(79,9 \%)$ podem ser prescritos em talonário de controle especial (Tabela 8).

Tabela 8. Distribuição dos alunos do Curso de Odontologia do Centro Universitário de João Pessoa (UNIPE) segundo o tipo de medicamento que pode ser prescrito em talonário especial

\begin{tabular}{l|c|c}
\hline \multirow{2}{*}{$\begin{array}{c}\text { Medicamentos que o cirurgião- } \\
\text { dentista pode prescrever em } \\
\text { talonário de controle especial }\end{array}$} & \multicolumn{2}{|c}{ Frequência } \\
\cline { 2 - 3 } & $\mathbf{n}$ & $\mathbf{\%}$ \\
\hline Analgésicos & 1 & 0,6 \\
\hline Ansiolíticos & 1 & 0,6 \\
\hline Antibióticos & 1 & 79,9 \\
\hline $\begin{array}{l}\text { Antibióticos, analgésicos e } \\
\text { antidepressivos }\end{array}$ & $\mathbf{0 , 6}$ \\
\hline Antibióticos e analgésicos & 7 & 1,3 \\
\hline Antibióticos e ansiolíticos & 4 & 2,6 \\
\hline Antibióticos e antidepressivos & 8 & 5,0 \\
\hline Antibióticos e anti-inflamatórios & 1 & 0,6 \\
\hline Antibióticos e controlados & 4 & 2,6 \\
\hline $\begin{array}{l}\text { Antibióticos, analgésicos e } \\
\text { anti-inflamatórios }\end{array}$ & 1 & 0,6 \\
\hline Antibióticos, tyles e codeína & 2 & 1,3 \\
\hline Anti-inflamatórios & 1 & 0,6 \\
\hline Psicotrópicos & $\mathbf{1 5 9}$ & $\mathbf{1 0 0}$ \\
\hline Total & \multicolumn{2}{|c}{}
\end{tabular}


Segundo a Agencia Nacional de Vigilancia Sanitária (ANVISA) ${ }^{17}$, conforme disposto no artigo 38 e artigo 55, parágrafo $1^{\circ}$ da Portaria SVS/MS $n^{\circ}$. $344 / 98$, o cirurgião-dentista pode prescrever quaisquer substâncias e medicamentos sujeitos a controle especial quando para uso odontológico. Assim, ao cirurgião-dentista é permitida a prescrição das substâncias relacionadas nas listas A1, A2, A3, B1 e C1.

Cerca de $80 \%$ dos alunos do Centro Universitário de João Pessoa (UNIPÊ) não conheciam a lista do RENAME (Tabela 9). Enquanto que a OMS recomenda que $100 \%$ das prescrições sejam realizadas a partir da lista de medicamentos essenciais (RENAME). Estudo conduzido por Farias et al. ${ }^{18}$ apontou que $91,9 \%$ dos medicamentos prescritos nas unidades básicas de Saúde da Família no município de Campina Grande (PB) seguiram a Relação Nacional de Medicamentos Essenciais (RENAME). Colombo et al. ${ }^{20}$ avaliaram amostragem do Programa de Saúde da Família de Blumenau (SC) e observaram que $57,7 \%$ dos medicamentos prescritos faziam parte da (RENAME).

Tabela 9. Distribuição dos alunos do Curso de Odontologia do Centro Universitário de João Pessoa (UNIPE) segundo o conhecimento em Relação Nacional de Medicamentos Essenciais (RENAME)

\begin{tabular}{l|c|c}
\hline \multirow{2}{*}{$\begin{array}{c}\text { Conhece o } \\
\text { RENAME? }\end{array}$} & $\mathbf{2}$ & $\mathbf{2}$ \\
\cline { 2 - 3 } & 30 & $\mathbf{1 8 , 9}$ \\
\hline Sim & 129 & 81,1 \\
\hline Não & $\mathbf{1 5 9}$ & $\mathbf{1 0 0}$ \\
\hline Total & \multicolumn{2}{|c}{}
\end{tabular}

Dentre os estudantes de Odontologia do Centro Universitário de João Pessoa (UNIPÊ), 86,2\% desconheciam os medicamentos pertencentes à lista do RENAME; os que relataram conhecer, não apontaram corretamente os medicamentos contidos na lista (Tabela 10).

Segundo a Agência Nacional de Vigilância Sanitária (ANVISA) ${ }^{19}$ dos medicamentos propostos só o diclofenaco de sódio, amoxicilina, dipirona, paracetamol e Transamin pertencem à lista do RENAME.

Quando questionados sobre o conhecimento quanto à prescrição de medicamentos injetáveis, $68,6 \%$ dos acadêmicos de Odontologia do Centro Universitário de João Pessoa (UNIPÊ) relataram conhecer essas drogas (Tabela 11).

Segundo Grou, et al. ${ }^{20}$ os profissionais envolvidos na prescrição, preparo e administração de medicamentos estão relativamente acostumados a administrar certos medicamentos em uma determinada via e, na vigência de alterações, surgem dúvidas e incertezas, principalmente quanto à via de administração intramuscular (IM) de medicamentos, o que demonstra a insegurança quanto à técnica de administração IM. As dúvidas surgidas, então, são quanto ao local a ser administrado, o músculo a ser atingido, bem como suas vantagens e desvantagens.

Tabela 10. Distribuição dos alunos do Curso de Odontologia do Centro Universitário de João Pessoa (UNIPE) segundo os medicamentos contidos na lista do RENAME

\begin{tabular}{|c|c|c|}
\hline \multirow{2}{*}{ Lista do RENAME } & \multicolumn{2}{|c|}{ Frequência } \\
\hline & $\mathbf{n}$ & $\%$ \\
\hline Amoxicilina & 1 & 0,6 \\
\hline Amoxicilina e Dipirona & 1 & 0,6 \\
\hline $\begin{array}{l}\text { Diclofenaco de sódio, amoxicilina e } \\
\text { paracetamol }\end{array}$ & 1 & 0,6 \\
\hline $\begin{array}{l}\text { Diclofenaco de sódio, amoxicilina, } \\
\text { dipirona e paracetamol }\end{array}$ & 5 & 3,0 \\
\hline $\begin{array}{l}\text { Diclofenaco de sódio, amoxicilina, } \\
\text { dipirona, paracetamol e buscopan }\end{array}$ & 1 & 0,6 \\
\hline $\begin{array}{l}\text { Diclofenaco de sódio, amoxicilina, } \\
\text { paracetamol e transamin }\end{array}$ & 1 & 0,6 \\
\hline $\begin{array}{l}\text { Diclofenaco de sódio, dipirona, } \\
\text { paracetamol e buscopan }\end{array}$ & 1 & 0,6 \\
\hline Não sei & 136 & 86,2 \\
\hline Nimesulida e Amoxicilina & 1 & 0,6 \\
\hline Nimesulida e Paracetamol & 1 & 0,6 \\
\hline $\begin{array}{l}\text { Nimesulida, diclofenaco de sódio, } \\
\text { amoxicilina, dipirona e paracetamol }\end{array}$ & 6 & 3,6 \\
\hline $\begin{array}{l}\text { Nimesulida, amoxicilina, dipirona } \mathrm{e} \\
\text { paracetamol }\end{array}$ & 3 & 1,8 \\
\hline Psicotrópicos & 1 & 0,6 \\
\hline Total & 159 & 100 \\
\hline
\end{tabular}

Tabela 11. Distribuição dos alunos do Curso de Odontologia do Centro Universitário de João Pessoa (UNIPE) segundo o conhecimento quanto à prescrição de medicamentos injetáveis

\begin{tabular}{l|c|c}
\hline \multirow{2}{*}{$\begin{array}{c}\text { Prescrição de } \\
\text { Injetáveis }\end{array}$} & $\mathbf{2}$ & Frequência \\
\cline { 2 - 3 } & 121 & 68,6 \\
\hline Sim & 38 & 21,4 \\
\hline Não & $\mathbf{1 5 9}$ & $\mathbf{1 0 0}$ \\
\hline Total &
\end{tabular}

Fonte: Dados da pesquisa.

Nessa pesquisa $83,6 \%$ dos estudantes de Odontologia do Centro Universitário de João Pessoa (UNIPÊ) apontaram a necessidade de apenas duas vias, $10,7 \%$ relataram a necessidade de três vias e $5,7 \%$ acreditam que apenas uma via de prescrição de receituário de controle especial seja necessária (Tabela 12).

Silva ${ }^{21}$ sugere que para receituário normal é necessária a confecção desse documento em duas vias, uma entregue ao paciente e outra anexada ao prontuário ele ainda acrescenta, que nos casos de prescrição em talonário de controle especial, a dispensa do medicamento somente poderá ser efetuada mediante receita, sendo, assim, obrigatoriamente em duas vias (uma ficará retida no estabelecimento farmacêutico e outra, devidamente carimbada pela farmácia, ficará com o paciente como comprovante do atendimento), sendo recomendada ainda uma terceira via para arquivamento junto ao prontuário.

De acordo com a distribuição da quantidade de medicamentos de controle especial, 55,3\% dos estudantes de Odontologia do Centro Universitário de João Pessoa (UNIPÊ) relataram que apenas um 
medicamento deveria ser prescrito, seguido de dois medicamentos com $20,1 \%$, três medicamentos com $10,7 \%$ e quantos o cirurgião-dentista achar necessário para 12,8\% (Tabela 13).

Segundo a Agencia Nacional de Vigilancia Saniária (ANVISA) ${ }^{17}$ não há quantidade limite de prescrição para o número de substâncias que podem ser prescritas na mesma receita, mas sim limitação pelo tempo de tratamento.

Tabela 12. Distribuição dos alunos do Curso de Odontologia do Centro Universitário de João Pessoa (UNIPE) segundo a quantidade de vias de prescrição de medicamentos de controle especial.

\begin{tabular}{l|c|c}
\hline \multirow{2}{*}{$\begin{array}{l}\text { Vias de prescrição } \\
\text { (controle especial) }\end{array}$} & $\mathbf{n}$ & Frequência \\
\cline { 2 - 3 } Uma & 9 & 5,7 \\
\hline Duas & 133 & 83,6 \\
\hline Três & 17 & 10,7 \\
\hline Total & $\mathbf{1 5 9}$ & $\mathbf{1 0 0}$ \\
\hline Fonte: Dados da pesquisa. & \multicolumn{2}{|}{}
\end{tabular}

Tabela 13. Distribuição dos alunos do Curso de Odontologia do Centro Universitário de João Pessoa (UNIPE) segundo a quantidade de medicamentos prescritos em talonário especial

\begin{tabular}{l|c|c}
\hline \multirow{2}{*}{$\begin{array}{c}\text { Prescrição de } \\
\text { medicamentos de } \\
\text { controle especial }\end{array}$} & $\mathbf{n}$ & $\mathbf{\%}$ \\
\cline { 2 - 3 } & 88 & 55,3 \\
\hline Um & 32 & 20,1 \\
\hline Dois & 18 & 10,7 \\
\hline Três achar & 21 & 12,8 \\
\hline $\begin{array}{l}\text { Quantos } \\
\text { necessário }\end{array}$ & $\mathbf{1 5 9}$ & $\mathbf{1 0 0}$ \\
\hline Total &
\end{tabular}

No presente estudo foi dado aos alunos do Centro universitário de João Pessoa (UNIPÊ) um caso clinico que relata paciente do gênero feminino, 20 anos de idade, aalérgica a diclofenaco de sódio, que compareceu a clinica escola de odontologia professor Afonso Pereira do Centro Universitário de João Pessoa (UNIPÊ) para avaliação odontológica, com diagnóstico de pericoronarite leve, sem sinais de infecção, sendo prescrito analgésico e antiinflamatório para alívio da dor. Frente ao caso clínico apresentado, os estudantes do Centro universitário de João Pessoa (UNIPÊ) foram questionados sobre o esquema farmacológico mais adequado . Entre as respostas colhidas a dipirona e o ibuprofeno ficaram em primeiro lugar com 51,6\% das respostas, seguido de paracetamol e diclofenaco de potássio com 32,1\%, amoxicilina, paracetamol e ibuprofeno e amoxicilina + clavulanato de potássio e ibuprofeno ambos com 8,2\% (Tabela 14).

Segundo Ferreira ${ }^{22}$ no caso de pericoronarite leve recomenda-se a aplicação tópica de peroxido de hidrogenio, ou entao digluconato de clorexidina $0,12 \%$, acompanhada de otima higienização até a diminuição do processo. A prescrição de analgesicos e antiinflamatórios, só se justificaria nos casos de pericoronarite grave, associada à antibioticoterapia por sete dias. Sendo a melhor solução para a prevenção e sua recidiva é a extração do terceiro molar envolvido.

Tabela 14. Distribuição dos alunos do Curso de Odontologia do Centro Universitário de João Pessoa (UNIPE) segundo esquema terapêutico para o primeiro caso clínico proposto

\begin{tabular}{l|c|c}
\hline \multirow{2}{*}{ Caso Clínico I } & \multicolumn{2}{c}{ Frequência } \\
\cline { 2 - 3 } & $\mathbf{n}$ & $\mathbf{\%}$ \\
\hline $\begin{array}{l}\text { Amoxicilina + clavulanato de } \\
\text { potássio + paracetamol }\end{array}$ & 13 & 8,2 \\
\hline Dipirona + Ibuprofeno & 82 & 51,6 \\
\hline $\begin{array}{l}\text { Paracetamol + Diclofenaco de } \\
\text { Potássio }\end{array}$ & 51 & 32,1 \\
\hline $\begin{array}{l}\text { Amoxicilina + Paracetamol + } \\
\text { Ibuprofeno }\end{array}$ & 13 & 8,2 \\
\hline Total & $\mathbf{1 5 9}$ & $\mathbf{1 0 0}$ \\
\hline Fonte: Dados da pesquisa. & \multicolumn{2}{|}{}
\end{tabular}

O segundo caso clinico apresentado aos alunos do Centro universitário de João Pessoa (UNIPÊ) fazia referência a paciente do gênero masculino, 40 anos de idade, que compareceu ao serviço de urgência do centro de especialidades odontológica com sinais flogísticos na região maxilofacial. Quando realizado o exame clinico extraoral foi observado edema em região infraorbitário direito causando oclusão palpebral e ao exame intraoral foi observada extensa destruição coronária do elemento 13 com indicação de exodontia. Depois do caso exposto foi perguntado qual o tratamento farmacológico proposto após a remoção do elemento, sabendo-se que o paciente é alérgico ao grupo das penicilinas. 59,1\% dos alunos relataram que o melhor tratamento farmacológico proposto seria a dipirona sódica, diclofenco de sódio, eritromicina e metronidazol.

Segundo a Agencia Nacional de Vigilância Sanitária (ANVISA) ${ }^{19}$ o tratamento farmacológico proposto nesses casos são os medicamentos constantes na lista do RENAME, tais como dipirona e diclofenaco, mas relata que para pacientes alérgicos ao grupo das penicilinas os antibióticos mais utilizados são a claritromicina, azitromicina e a eritromicina.

Tabela 15. Distribuição dos alunos do Curso de Odontologia do Centro Universitário de João Pessoa (UNIPE) segundo esquema terapêutico para o segundo caso clínico proposto

\begin{tabular}{l|c|c}
\hline \multirow{2}{*}{ Caso Clínico II } & \multicolumn{2}{|c}{ Frequência } \\
\cline { 2 - 3 } & $\mathbf{n}$ & $\mathbf{\%}$ \\
\hline $\begin{array}{l}\text { Dipirona, ibuprofeno, } \\
\text { amoxicilina e metronidazol }\end{array}$ & 21 & 13,2 \\
\hline $\begin{array}{l}\text { Paracetamol, diclofenaco, } \\
\text { gentamicina e amoxicilina }\end{array}$ & 10 & 6,3 \\
\hline $\begin{array}{l}\text { Codeína, nimesulida, } \\
\text { cefalexina e cefalotina }\end{array}$ & 34 & 20,8 \\
\hline $\begin{array}{l}\text { Dipirona, diclofenaco de } \\
\text { sódio, eritromicina e } \\
\text { metronidazol }\end{array}$ & 94 & 59,1 \\
\hline Total & $\mathbf{1 5 9}$ & $\mathbf{1 0 0}$ \\
\hline
\end{tabular}

Fonte: Dados da pesquisa. 


\section{CONCLUSÃO}

Consideramos que é baixo o nível de conhecimento dos estudantes de odontologia do Centro Universitário de João Pessoa - UNIPÊ em relação a os medicamentos contidos na lista de medicamentos essenciais (RENAME) do Sistema Único de Saúde (SUS), tendo em vista que muitos dos estudantes irão atuar nos postos da estratégia da saúde da família, local esse onde, a maioria dos pacientes possui uma renda mensal baixa, impedindo assim a aquisição de medicamentos que não sejam disponíveis gratuitamente levando a o não cumprimento dos horários propostos para tomar suas medicações;

Os estudantes em sua maioria relataram se sentirem seguros em relação a prescreverem para seus pacientes, tendo a maior parte deles participados de cursos extracurriculares.

Segundo os casos clínicos foi razoável o nível de conhecimento dos estudantes de odontologia do centro universitário de João Pessoa - UNIPÊ em relação à prescrição de medicamentos para os pacientes, pois um pouco mais da metade acertaram os casos propostos.

\section{REFERÊNCIAS}

1. Sharif, S. I.; Al-shaqra, M.; Hajjar, H.; Shamout, A.; Wess, L. Patterns of drug prescribing in a hospital in Dubai, United Arab Emirates (UAE). Lybian J Med. 2008;3(1):10-12.

2. Vieira FS. Assistência farmacêutica no sistema público de saúde no Brasil. Rev Panam Salud Pública. 2010;27(2):149-56.

3. Bertoldi AD, Barros AJD, Camargo AL, Hallal PC, Vandoros S, Wagner A et al. Household expenditure for medicines and the role of free medicines in the Brazilian public health system. Am J Public Health. 2001;101(5):916-21.

4. Arrais PSD, Brito LL, Barreto ML, Coelho HLL. Prevalência e fatores determinantes do consumo de medicamentos no Município de Fortaleza, Ceará, Brasil. Cad Saúde Pública. 2005;21(6):1737-46.

5. Garcia ANM, Romani SAM, Lira PIC. Indicadores antropométricos na avaliação nutricional de idosos: um estudo comparativo. Rev Nutr. 2007;20(4):371-78.

6. Silva EL, Menezes EM. Metodologia da Pesquisa e elaboração de dissertação. 4. ed. Florianópolis: Editora UFSC; 2005.

7. Gil AC. Como elaborar projetos de pesquisa. São Paulo. Atlas; 1991.

8. Britto TA, Castilho LS, Paixão HH. Os estudantes de odontologia e a (in) segurança para prescrever medicamentos. Arq Centro Estud Curso de Odontol. 1996;32(1):51-64.

9. Araújo LG, Biagini FC, Fernandes RL, Caputo IGC, Silva RHA. Conhecimento de acadêmicos de
Odontologia sobre os aspectos clínicos, éticos e legais da prescrição medicamentosa. RFO Passo Fundo. 2012;17(1):50-4.

10.Costa SM, Durães SJA, Abreu MHNG. Feminização do curso de odontologia da Universidade Estadual de Montes Claros. Rev. Ciênc saúde coletiva. 2010;15(suppl 1):1865-73.

11.Sundefeld MLMM, Perri SHV, Borghi WMMC, Rodrigues MAB. Escolhendo a profissão: opinião de alunos de odontologia e medicina veterinária. Omnia saúde. 2011;8(1):36.

12.Brustolin J, Brustolin J, Toassi RFC, Kuhnen M. Perfil do acadêmico de odontologia da Universidade do Planalto Catarinense - Lages SC, Brasil. Rev ABENO. 2006;6(1):70-76.

13. Sullcahuamán JAG. Fatores associados à qualidade de vida relacionada à saúde bucal em estudantes de odontologia [dissertação]. Curitiba: Universidade Federal do Paraná; 2012.

14.Castilho LS, Paixâo HH, Perini E. Prescrição de medicamentos de uso sistêmico por cirurgiõesdentistas clínicos gerais. Rev Saúde Pulica. 1999;33(3):287-94.

15.Marmitt GF. Prescrição de medicamentos para tratamento da dor por cirurgiões-dentistas vinculados a uma faculdade de odontologia [trabalho de conclusão de curso]. Porto Alegre: Universidade Federal do Rio Grande do Sul; 2010.

16. Garbin CAS, Garbin AJI, Rovida TAS, Moroso TT, Dossi AP. Conhecimento sobre prescrição medicamentosa entre alunos de Odontologia: o que sabem os futuros profissionais? Rev Odontol Unesp. 2007;36(4):323-32.

17.Agência Nacional de Vigilância Sanitária (ANVISA). 2018. Medicamentos controlados: perguntas frequentes. Disponível em: http://portal.anvisa.gov.br/medicamentos/pergunta s-frequentes. Acesso em: 05 maio 2018.

18.Farias AD, Cardoso MAA, Medeiros ACD, Belém LF, Simões MOS. Indicadores de prescrição médica nas unidades básicas de Saúde da Família no município de Campina Grande, PB. Rev Bras Epidemiol. 2007;10(2):149-56.

19. Agencia Nacional de Vigilanca Sanitária (ANVISA). 2018. Antimicrobianos - bases teóricas e uso clínico. Disponível em: http://portal.anvisa.gov.br/medicamentos/pergunta s-frequentes. Acesso em: 05 maio 2018.

20. Grou CR, Miasso AI, Cassiani SHB, Silva AEBC, Fakih FT. Erros de medicação: tipos, fatores causais e providências tomadas em quatro hospitais brasileiros. Rev Esc Enferm USP. 2006;40(4):524-32.

21.Silva RHA. Orientação Profissional para o cirurgião-dentista: ética e legislação. São Paulo: Santos; 2010. 
22.Ferreira PM. complicações pós operatórias de terceiros molares inclusos [Trabalho de conclusão de curso]. Piracicaba: Universidade Estadual de Campinas (UNICAMP) Faculdade de Odontologia de Piracicaba; 2004.

\section{CONFLITO DE INTERESSES}

Os autores declaram não haver conflitos de interesse.

AUTOR PARA CORRESPONDENCIA

Larissa Suelen da Silva Lins

larissass1@hotmail.com

Submetido em 17/08/2018

Aceito em 04/10/2019 